This document is confidential and is proprietary to the American Chemical Society and its authors. Do not copy or disclose without written permission. If you have received this item in error, notify the sender and delete all copies.

\title{
Multipolar Force Fields and Their Effects on Solvent Dynamics Around Simple Solutes
}

\begin{tabular}{|r|l|}
\hline Journal: & The Journal of Physical Chemistry \\
\hline Manuscript ID: & jp-2014-08052q.R2 \\
\hline Manuscript Type: & Article \\
\hline Date Submitted by the Author: & 11 -Jan-2015 \\
\hline Complete List of Authors: & $\begin{array}{l}\text { Jakobsen, Sofie; Aarhus University, Department of Chemistry } \\
\text { Bereau, Tristan; Max Planck Institute for Polymer Research, } \\
\text { Meuwly, Markus; University of Basel, Department of Chemistry }\end{array}$ \\
\hline
\end{tabular}

SCHOLARONE

Manuscripts 


\title{
Multipolar Force Fields and Their Effects on Solvent
} Dynamics Around Simple Solutes

\author{
Sofie Jakobsen, ${ }^{*, \dagger, \dagger}$ Tristan Bereau, ${ }^{\dagger}, \uparrow$ and Markus Meuwly ${ }^{*, \dagger}$ \\ Department of Chemistry, University of Basel, Klingelbergstrasse 80, 4056 Basel, \\ Switzerland, and Department of Chemistry, Aarhus University, Langelandsgade 140, 8000 \\ Aarhus C, Denmark \\ E-mail: sj@chem.au.dk; m.meuwly@unibas.ch
}

\begin{abstract}
The performance of multipole (MTP) and point charge (PC) force fields in classical molecular dynamics (MD) simulations of condensed-phase systems for both equilibrium and dynamical quantities is compared. MTP electrostatics provides an improved description of the anisotropic electrostatic potential, which is especially important to describe key, challenging interactions, such as lone pairs, $\pi$-interactions, and hydrogen bonds. These chemical environments are probed by focusing on the hydration properties of two molecules: $N$-methylacetamide and phenyl bromide. Both, equilibrium and dynamical quantities are affected by the quality of the electrostatic model. The alteration of the first solvation shell in the MTP simulations is validated by comparing with lifetimes and correlation times of solute-solvent interactions from experiment. The improved dynamical behavior found in the MTP simulations - observed for molecules
\end{abstract}

\footnotetext{
* To whom correspondence should be addressed

${ }^{\dagger}$ Department of Chemistry, University of Basel, Klingelbergstrasse 80, 4056 Basel, Switzerland

${ }^{\ddagger}$ Department of Chemistry, Aarhus University, Langelandsgade 140, 8000 Aarhus C, Denmark

IPresent address: Max Planck Institute for Polymer Research, Ackermannweg 10, 55128 Mainz, Germany 
parametrized using very different protocols — suggests that a systematic improvement of both equilibrium and dynamical quantities when using MTP electrostatics is possible.

\section{Introduction}

All-atom simulations of large molecules over long time scales usually rely on an empirical expression for the intermolecular interactions, i.e. a force field. The force field energy is conveniently decomposed into bonded and non-bonded terms, which are parametrized from ab initio or experimental results. ${ }^{1}$ The electrostatic term often dominates intermolecular interactions, in particular for polar systems, making the electrostatic energy an important target for a realistic description of the interactions. The electrostatic energy function is usually parametrized in terms of PCs, even though this model is not sufficiently flexible to account for certain features of the complex molecular charge distribution. Improvements to standard force fields have been proposed, including the use of atomic MTPs and atomic polarizabilities. ${ }^{2-6}$ The anisotropic nature of MTPs allows for a more accurate representation of the static electrostatic potential (ESP), while polarizabilities describe variations in the ESP due to fluctuations in the environment, such as solvent effects.

The improvement of the static ESP from higher order multipole moments is widely documented in the literature, ${ }^{7-13}$ but it may be argued that off-site atomic charges provide a comparable improvement in representing the electric field. The OPLS-AAx FF includes partial positive charges along the $\mathrm{C}$-X axis of halogen bonds, ${ }^{14}$ and in the TIP5P water model $^{15}$ additional charges are used at the lone-pair sites of oxygen to improve the model. However, addition of off-site charges requires optimization of both placement and magnitude

of the charges, and the issue of rank deficiency will make this a non-trivial task. ${ }^{16,17}$ On the other hand, atomic MTPs provide a natural framework for improving force field electrostatics beyond the PC approximation. Stone has pioneered the derivation and use of MTPs to 
study intermolecular interactions, ${ }^{18}$ and has formulated the distributed multipole analysis (DMA), ${ }^{19,20}$ which is one of several methods to determine a set of nonunique atomic MTPs based on electronic structure calculations. Other notable schemes include the Cumulative Atomic Multipole Moments (CAMM) ${ }^{21,22}$ and Atoms in Molecules (AIM). ${ }^{23}$

The AMOEBA force field is an example of a force field going beyond the PC approximation by applying both permanent atomic MTPs up to quadrupoles as well as atomic polarizabilities in terms of induced dipoles. ${ }^{5,24}$ The improved performance of the AMOEBA force field compared to standard atomistic models is due to both permanent MTPs and polarizabilities, with a study of conformational energies suggesting the two effects are of similar importance. ${ }^{25}$ The use of higher order moments requires the definition of a local coordinate frame of each atom, which can be done in several ways. AMOEBA uses the 'z-then-bisector' local frame definition, ${ }^{24}$ where axes are placed along major chemical determinants, in order to follow the movement of vibrations and rotations. In the present work we use the local axis framework described by Kramer et al., ${ }^{11}$ where the axes are preferentially aligned along major axes of symmetry to minimize the number of non-zero MTP components. ${ }^{26}$

While MTPs are known to better describe the ESP of small (diatomic) and medium-sized molecules, their advantage in accurately computing intermolecular interactions was found to be only minor for representative systems including benzonitrile or formamide. ${ }^{27}$ As an example, when using ESP-optimized PCs or MTPs on several thousand random conformations for benzonitrile (BZN) and formamide (FAD) homodimers, the root mean squared error relative to $\mathrm{MP} 2 / 6-311+\mathrm{G}^{*}$ reference calculations was virtually identical (0.21 and 0.22 $\mathrm{kcal} / \mathrm{mol}$ for BZN and 0.50 and $0.49 \mathrm{kcal} / \mathrm{mol}$ for FAD). ${ }^{27}$ In the present work we extend and scrutinize the utility of MTP-based electrostatics for dynamical properties by considering the solvation dynamics of two important and chemically different model compounds: $N$-methylacetamide (NMA) and phenyl bromide (PhBr). Until now, the effects of using PC- 
and MTP-electrostatics on dynamical properties have not been studied extensively, specifically in comparison with experimental data. Attempts to explicitly assess the difference in performance between PC- and MTP-based electrostatics include the pocket-dynamics of diatomic molecules in myoglobin, ${ }^{28}$ vibrational relaxation and spectroscopy of $\mathrm{CN}^{-}$in aqueous solution, ${ }^{29,30}$ and the two-dimensional spectroscopy of NMA. ${ }^{31}$

All-atom MD simulations provide the necessary information to characterize the inter- and intramolecular dynamics in solute-solvent systems. By comparing with experimentally accessible information, e.g., water residence and reorientation times around the solute, one can assess and compare the performance of PC and MTP electrostatics models. For solvated cyanide it is found that MTP-based electrostatics leads to better agreement with experiment, and systematic improvements of the hydration dynamics regardless of the solute's MTP-based parametrization strategy. ${ }^{29,30,32}$ In the present work these considerations are extended to larger systems exhibiting different topical interactions with the surrounding solute. It is of particular interest to assess whether the improved description afforded by MTP over PC electrostatics - as judged from comparisons to results from quantum chemical calculations - also extends to improved observables from explicit MD simulations and comparison with experiment.

\section{Computational Methods}

\section{Force field parametrization}

The ESP, $\phi$, at a point $\mathbf{r}$ arising from $M$ nuclei and the electron density $\rho$ is given by

$$
\phi(\mathbf{r})=\sum_{i}^{M} \frac{Z_{i}}{\left|\mathbf{R}_{i}-\mathbf{r}\right|}-\int \frac{\rho\left(\mathbf{r}^{\prime}\right)}{\left|\mathbf{r}^{\prime}-\mathbf{r}\right|} \mathrm{d} \mathbf{r}^{\prime}
$$


Here, $\mathbf{R}_{i}$ and $Z_{i}$ are the position and charge of nucleus $i$, respectively. Following the notation of Stone, ${ }^{18}$ a Taylor expansion around each nucleus $1 / R_{i} \equiv 1 /\left|\mathbf{R}_{i}-\mathbf{r}\right|$ provides the following distributed multipole expansion of the ESP in atomic units

$$
\phi(\mathbf{r})=\sum_{i=1}^{M}\left[\frac{q_{i}}{R_{i}}+\frac{\mu_{i, \alpha} R_{i, \alpha}}{R_{i}^{3}}+\frac{1}{3} \Theta_{i, \alpha \beta} \frac{3 R_{i, \alpha} R_{i, \beta}-R_{i}^{2} \delta_{\alpha \beta}}{R_{i}^{5}}+\ldots\right]
$$

where $i$ labels an atom with charge $q_{i}$, a dipole moment with the Cartesian $\alpha$-components $\mu_{i, \alpha}$, and a quadrupole moment with Cartesian $\alpha \beta$-components $\Theta_{i, \alpha \beta}$. The Einstein summation convention is applied.

Atomic MTPs up to quadrupoles are oriented using suitable local reference axis systems. ${ }^{11}$ The MTPs are optimized in a least-squares fit to reproduce the ESP from MP2/cc-pVDZ calculations. The fit includes the first interaction belt, i.e., the range $1.66 \sigma<r<2.2 \sigma$, where $r$ is the distance from any nucleus and $\sigma$ is the atomic site's vdW radius. ${ }^{33}$ To assess the bias exerted by the force-field parametrization, we followed distinct strategies for the two molecules. The PC parametrization for NMA, obtained from CGenFF ${ }^{34,35}$ was augmented to include MTP coefficients. To ensure that the MTP force field was a correction of the original one, monopoles were restrained to a small deviation (i.e., 0.005e), naturally damping the strength of the higher MTPs. This is the MTPW parametrization as described by Cazade et al. ${ }^{31}$ On the other hand, the parametrization of $\mathrm{PhBr}$ was more loosely based on its $\mathrm{PC}$ counterpart: a larger deviation of the PC-based monopoles (i.e., 0.1e) allowed more variability between the PC- and MTP-based force fields. ${ }^{36}$ In both cases, a reparametrization of the Lennard-Jones parameters was necessary to better reproduce experiments and for consistent vdW parameters, as described in earlier work. ${ }^{26}$ For both PC and MTP parametrizations we have employed the TIP3P water model. ${ }^{37}$ The final force fields were validated to reproduce thermodynamic quantities: pure-liquid densities, heats of vaporization, and hydration free energies. 


\section{Simulation protocol}

All simulations were performed in CHARMM $^{38}$ with the MTPL module ${ }^{36}$ for MTP electrostatics. Particle Mesh Ewald (PME) is used for the PC-PC interactions with grid-size spacing of $1 \AA$, relative tolerance of $10^{-6}$, and interpolation order 4 for long-range electrostatics, and a $12 \AA$ cutoff and $10 \AA$ switching for Lennard-Jones interactions. Only the solute carries higher order multipoles, resulting in only a slight increase in the computational cost for the MTP compared to the PC simulations. For more details on the simulation protocol and a discussion of the computational investment of MTP interactions, see Ref. 36.

NMA and PhBr are individually solvated in boxes of 882 and 515 TIP3P water molecules, respectively. The Hoover heat-bath method with pressure coupling ${ }^{39}$ at $T=298 \mathrm{~K}, p=1$ atm was used for the simulations, and the masses of the temperature and pressure piston was set to roughly $20 \%$ and $2 \%$ of the system's mass, respectively. The systems were first structurally relaxed using steepest descent minimization, then heated to $298 \mathrm{~K}$ at constant volume for $40 \mathrm{ps}$, followed by a 40 ps NPT equilibration using a Langevin damping coefficient on the piston $\gamma_{p}=20 \mathrm{ps}^{-1}$. The solvent distribution results are based on $10 \mathrm{~ns}$ of simulation, where frames are sampled every $500 \mathrm{fs}$, while the analysis of lifetimes and time correlation function is based on an additional set of $10 \mathrm{~ns}$ simulations for NMA and $5 \mathrm{~ns}$ simulations for $\mathrm{PhBr}$, both sampled every 10 fs. Lennard-Jones long-range corrections were applied to both the energy and the virial.

\section{Analysis}

Solvent distribution: The equilibrium properties of a liquid are commonly analyzed using the pair distribution function, $g(r)$. By its very nature, $g(r)$ averages over all directions, and thwarts any chance of observing subtle anisotropic features that we expect from MTP 
(a) For the hydrogen bond the definition from Luzar et al. ${ }^{40}$ is used which assigns a hydrogen bond for a minimum distance between the amide oxygen and the water hydrogen of $R_{\mathrm{OH}}<2.425 \AA$.

(b) The $\pi \cdots \mathrm{H}-\mathrm{O}$ coordination is defined by two cutoffs: the distance from a hydrogen to the center of the phenyl group projected to the $z$-axis, $R_{\mathrm{ph}, \mathrm{H}}^{z}$, and the same distance projected to the $x y$-plane, $R_{\mathrm{ph}, \mathrm{H}}^{x y}$. Prakash et al. ${ }^{41}$ have studied similar $\pi \cdots \mathrm{H}-\mathrm{O}$ interactions in a set of ab initio optimized benzene-water clusters. Based on this work, we choose the cutoff values sufficiently large to include both water hydrogens of the nearest water molecule in the set of 
geometries; $R_{\mathrm{ph}, \mathrm{H}}^{z}<4.0 \AA$ and $R_{\mathrm{ph}, \mathrm{H}}^{x y}<1.5 \AA$.

(c) For the $\sigma \cdots \mathrm{O}-\mathrm{H}$ interaction (also known as halogen bonding) we used the Br.. O distance projected onto the $x$-axis and the $y z$-plane, respectively. The cutoffs are chosen so that they enclose the region where it will be shown that the oxygen distribution varies the most between the PC and MTP electrostatics; $R_{\mathrm{Br}, \mathrm{O}}^{x}<3.5 \AA$ and $R_{\mathrm{Br}, \mathrm{O}}^{y z}<3.0 \AA$.

Solvent Dynamics: The hydrogen-bond fluctuation correlation function, $C_{n}(t)$ is calculated for NMA to analyze the time evolution of hydrogen bonds. It is constructed from the number $n(t)$ of water molecules that are hydrogen bonded to the $\mathrm{C}=\mathrm{O}$ group at time $t$ as follows:

$$
C_{n}(t)=\frac{\langle\delta n(0) \delta n(t)\rangle}{\langle\delta n(0)\rangle^{2}}
$$

Here, the numerator is the occupation-time correlation function (TCF), the denominator is the normalization, and $\delta n(t)=n(t)-\langle n\rangle$ is the fluctuation of the number of hydrogen bonds around its average over the entire trajectory, $\langle n\rangle . C_{n}(t)$ is calculated using an in-house code from which the hydrogen-bond fluctuation time $\tau_{\mathrm{H}}$ can be determined.

Rotational TCFs $C_{\mathrm{r}}(t)$ are calculated using CHARMM's CORREL utility. Only water molecules that are hydrogen bonded to the $\mathrm{C}=\mathrm{O}$ group for at least 10 ps are considered. However, since the hydrogen bond might break shortly and then rebind, we have used a larger cut-off, $R_{\mathrm{OO}}>5.0 \AA$, for which we consider the hydrogen bond as broken. For this set of water molecules the time correlation function of the torsion angle from the amide group

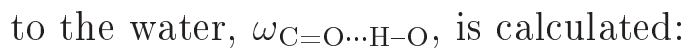

$$
C_{\mathrm{r}}(t)=\frac{\langle\omega(0) \omega(t)\rangle}{\langle\omega(0)\rangle^{2}}
$$

In the evaluation of $C_{\mathrm{r}}(t)$ we have correlated over the following 50 ps after the formation of the hydrogen bond. During the $10 \mathrm{~ns}$ simulations this provides $333 C_{\mathrm{r}}(t)$ functions for PC 
and 240 for MTP, from which the average and the standard deviation are calculated.

\section{Results}

In the following, results for NMA and $\mathrm{PhBr}$ are discussed separately.

\section{$N$-methylacetamide}

$N$-methylacetamide (NMA) is a convenient surrogate for a protein backbone and has thus been the subject of intense work, both computationally ${ }^{42-46}$ and experimentally, including infrared spectroscopy, ${ }^{47}$ two-dimensional infrared spectroscopy, ${ }^{48,49}$ and vibrational relaxation experiments. ${ }^{50,51}$ As a consequence, protein force-field parametrizations are often based on NMA. ${ }^{52-54}$ The $\mathrm{C}=\mathrm{O}$ group is a strong hydrogen-bond acceptor and allows one to study the hydrogen-bond solvent dynamics. For characterizing the environmental dynamics, solvent lifetimes and reorientation times for water molecules coordinated to the amide oxygen of NMA from simulations with PCs and MTPs are described and compared.

The ESP of NMA at the MP2/cc-pVDZ level is shown in Figure 1 (a), while those from the fitted PCs and MTPs are shown in Figures 1 (b) and (c). Projections onto the $x y$-plane which intersect the $\mathrm{C}=\mathrm{O}$ bond and the $N$-methyl carbon are reported. Throughout this work we report the strength of the ESP as an interaction energy (in units of $\mathrm{kJ} / \mathrm{mol}$ ) with a probe charge of 1 atomic unit $(1 e)$. The region inside the vdW radius of any atom is omitted, since the force field ESP is not meaningful close to the nuclei. Black contour lines are drawn at $1.66 \sigma$ and $2.2 \sigma$, which defines the region used for fitting MTPs. The mean absolute deviation (MAD) between the force field and the MP2 potential in the fitting region is 3.6 $\mathrm{kJ} / \mathrm{mol}$ for the PC parametrization and $3.2 \mathrm{~kJ} / \mathrm{mol}$ using MTPs. The small improvement in the ESP is a result of how the MTP parametrization was carried out: by designing an MTP force field in which the MTPs are corrections to the PCs, higher order MTPs will tend 


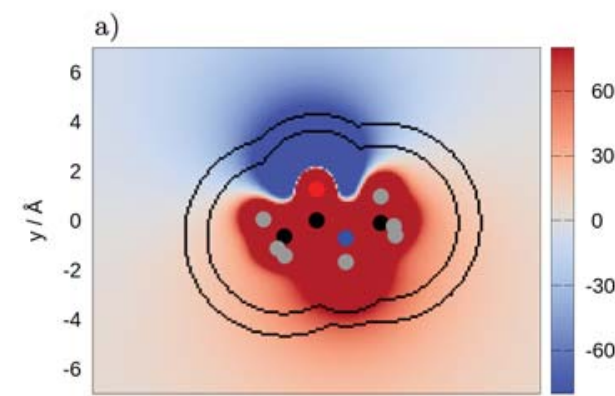

b)
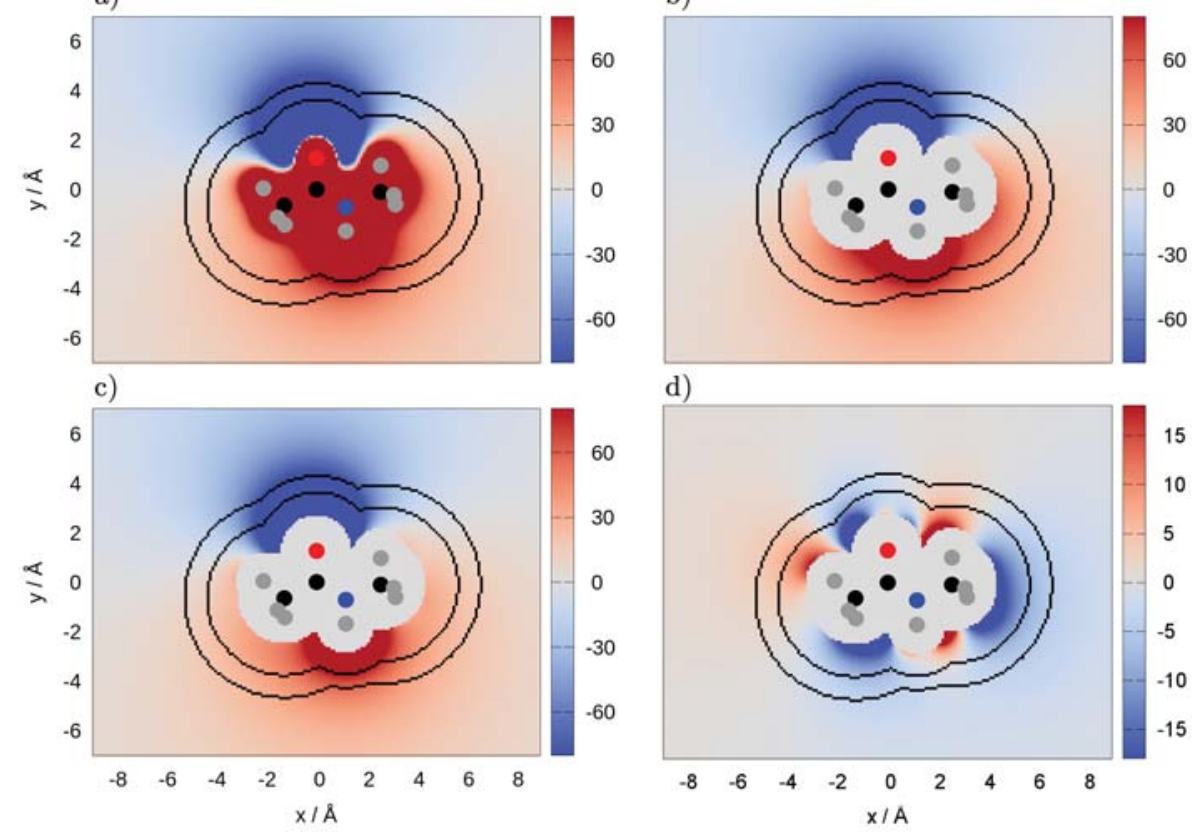

d)

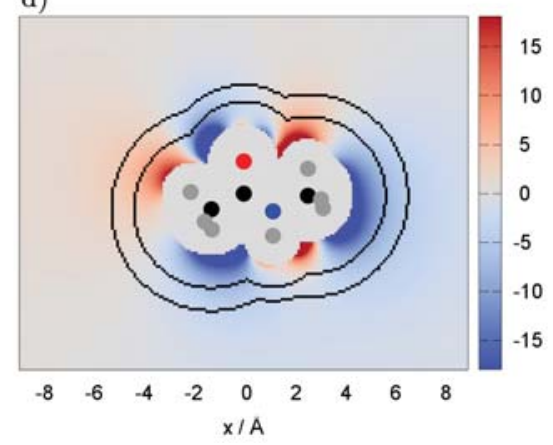

Figure 1: ESP of NMA in units of kJ/mol. a) MP2/cc-pVDZ, b) PC parametrization, c) MTP parametrization, and d) difference between MTP and PC $\left(\phi_{\mathrm{MTP}}-\phi_{\mathrm{PC}}\right)$. The black contour lines define the region used for fitting MTP parameters, corresponding to $1.66 \sigma$ and $2.2 \sigma$, respectively. 
to have a smaller impact on the ESP. In a full parametrization - i.e. without constraining the monopoles - an even lower MAD could be achieved. Figure 1 (d) shows the difference between the MTP and the PC parametrizations, $\phi_{\mathrm{MTP}}-\phi_{\mathrm{PC}}$, where red regions highlight regions in which the MTP potential is more positive than the PC potential, and vice versa for blue regions.

The difference between the PC and the MTP parametrizations is largest close to the nuclei where the solvent rarely enters, except when the solvent forms hydrogen bonds with the amide $\mathrm{C}=\mathrm{O}$ or $\mathrm{N}-\mathrm{H}$ groups. Previous simulations and experimental studies are primarily concerned with the dynamics around the $\mathrm{C}=\mathrm{O}$ group, ${ }^{31,43,49,50}$ and the analysis of NMA in the present study is therefore also focused on this. The PC and MTP models lead to a similarly negative $\mathrm{ESP}$ along the $\mathrm{C}=\mathrm{O}$ bond, but they differ in the potential angularly displaced from the $\mathrm{C}=\mathrm{O}$ bond axis (blue region of Figure $1(\mathrm{~d})$ ), where PCs predict a less negative potential compared to MTPs. This reflects the fact that MTPs are better suited to capture anisotropies in the electrostatic interactions than PCs. It should be noted, however, that ESPs do not necessarily reflect the overall quality of a force field. Even if the ESP is not reproduced perfectly, the final force field might still provide a meaningful description for different thermodynamic properties if, for example, the vdW term compensates for deficiencies in the electrostatic interactions.

For each snapshot in the MD simulation a local-axis framework was defined as described in the Method section, and the distribution of water hydrogens and oxygens were calculated on a two-dimensional grid in the $x z$-plane (i.e. the same plane used in Figure 1). The waterhydrogen distributions are shown in Figures 2 (a) and 2 (b) for PCs and MTPs, respectively. The difference between the two is given in Figure 2 (c), where blue regions correspond to increased solvent occupation in the PC parametrization compared to MTP, and vice versa for red regions. The corresponding oxygen distributions and difference of distributions are 

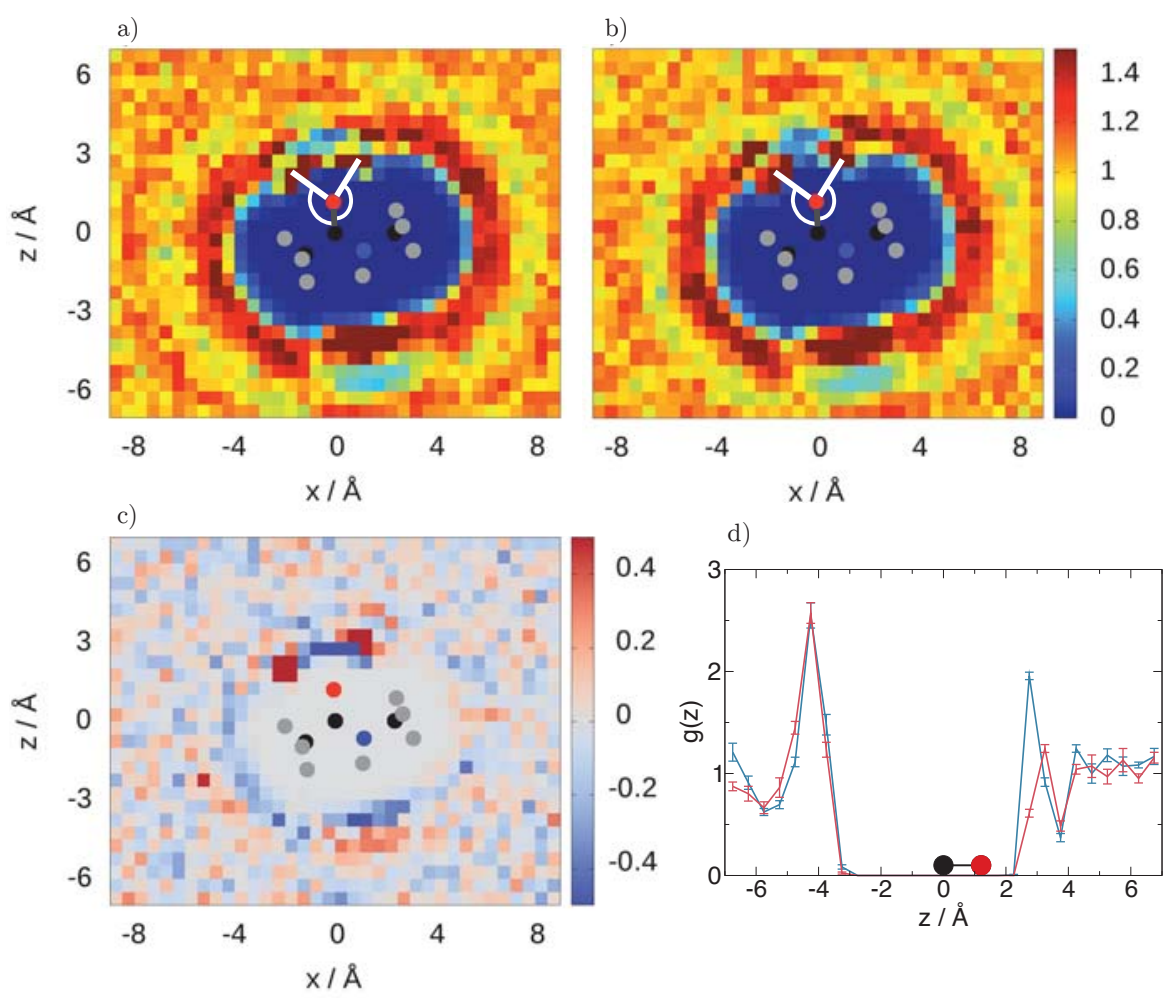

Figure 2: Two-dimensional pair distribution function $g(x, z)$ of water hydrogens projected onto the $x z$-plane intersecting NMA through the $\mathrm{C}=\mathrm{O}$ bond and the $N$-methyl carbon. Panels a) NMA parametrized with PCs and b) NMA parametrized with MTPs. White lines in a) and b) indicate the angle from the $\mathrm{C}=\mathrm{O}$ bond to the regions with highest density of hydrogens. Panels c) difference between MTPs and PCs, $g_{\mathrm{MTP}}-g_{\mathrm{PC}}$, and d) $1 \mathrm{D} g(z)$ for $x=0$ along the $\mathrm{C}=\mathrm{O}$ bond axis (atoms shown with dots) with $\mathrm{PC}$ shown in blue and MTP in red. The error bars correspond to $1 \sigma$ standard deviations calculated from five independent trajectories. 


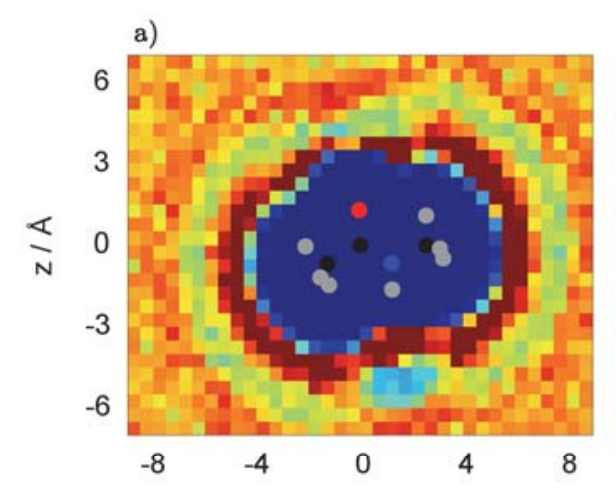

b)
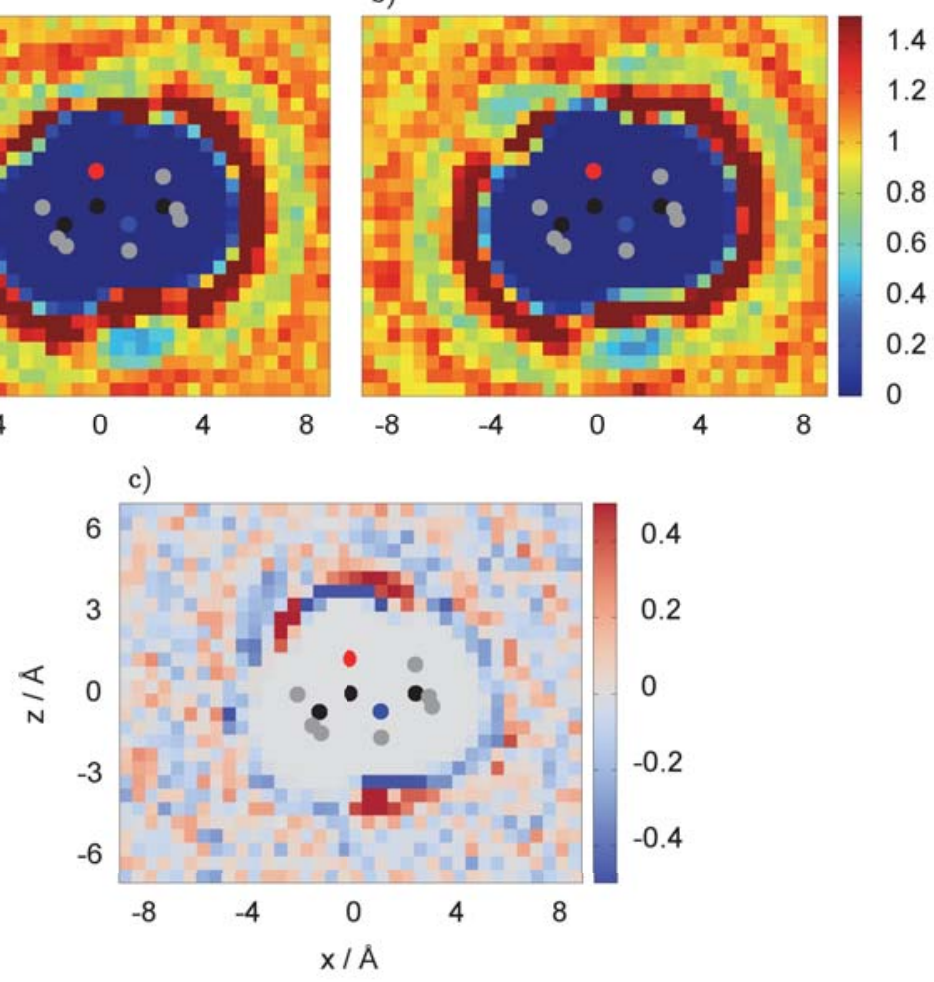

Figure 3: Two-dimensional pair distribution function $g(x, z)$ of water oxygen plotted on the $x z$-plane intersecting NMA through the $\mathrm{C}=\mathrm{O}$ bond and the $N$-methyl carbon a) parametrized with PCs. b) parametrized with MTPs. c) difference between MTPs and PCs, $g_{\mathrm{MTP}}-g_{\mathrm{PC}}$. 
reported in Figures 3 (a) to (c). The solvent pair-distribution functions are generalizations of $g(r)$ and have a limiting value of 1 at large distances, as seen from the (a) and (b) panels in Figure 2 and 3, because we report deviations from a homogeneous liquid.

For the hydrogen distribution, the largest difference between the PC and the MTP models are found in the region close to the amide oxygen, whereas for the water-oxygens, differences are large around the amide nitrogen as well. Figures 2 (a) and (b) show that water hydrogens close to the amide oxygen are most often found displaced from the $\mathrm{C}=\mathrm{O}$ bond axis. The two white lines at each side of the $\mathrm{C}=\mathrm{O}$ bond indicate this region. Figures 2 (c) and 3 (c) clearly show that the solvent distributions around the amide oxygen differs between the PC and MTP models. Furthermore, Figure $2(\mathrm{~d})$ shows a 1D plot of $g(z)$ where $x$ and $y$ are fixed to the values of the $\mathrm{C}=\mathrm{O}$ bond axis $(x=0 ; y=0)$ with error bars showing the standard deviation. The pair distribution function peaks in the first solvation shell at a value of 1.2 using PCs and 2.0 using MTPs, while the standard deviation in this region is less than 0.04 in both cases. Hence, the first solvation shell is significantly changed in occupation as a result of the different electrostatics. The standard deviations for the entire two-dimensional surface are of the same order of magnitude as in Figure 2 (d) and reported in Figures S1 and S2 in the Supporting Information.

In order to compare the orientation of the hydrogen bonds further, we analyze the distribution of the $\mathrm{C}=\mathrm{O}_{\mathrm{NMA}} \cdots \mathrm{H}_{\mathrm{w}}$ angle $\theta$ for the hydrogen-bonded water molecules, as defined in the Method section. Figure 4 shows the normalized distributions, $P(\theta)$. The two parametrizations yield comparable angles with maximum in $P(\theta) \sim 120^{\circ}$, but the distribution is narrower using MTPs, since the anisotropy allows for a more correct description of directional changes in the ESP. The angle of maximum hydrogen distribution around O in Figures 2 (a) and (b), indicated by the white lines, coincides with $\sim 120^{\circ}$. Furthermore, a computational study of NMA-water clusters optimized at the MP2 $/ 6-31++\mathrm{G}(\mathrm{d}, \mathrm{p})$ level ${ }^{55}$ reports a $\mathrm{C}=\mathrm{O} \cdots \mathrm{H}$ angle 
for four different local minima with $\theta$ ranging from $110^{\circ}$ to $130^{\circ}$, in good agreement with Figure 4.

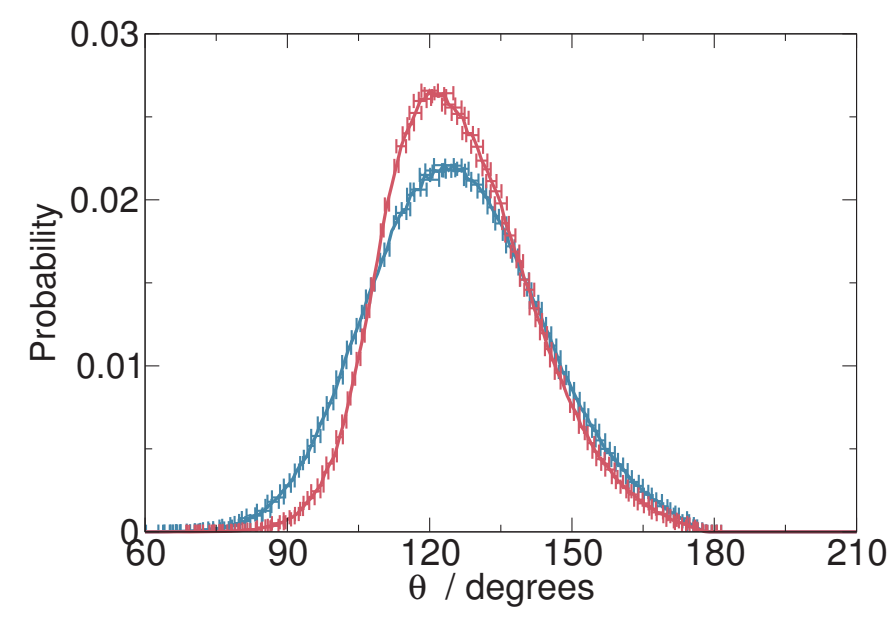

Figure 4: Distribution of the $\mathrm{C}=\mathrm{O} \cdots \mathrm{H}$ angle for water molecules that are hydrogen bonded to the amide oxygen of NMA for PC (blue) and MTP (red) shown with $1 \sigma$ standard deviations from the five independent runs of $1 \mathrm{~ns}$ each.

The fact that MTPs generate a narrower distribution in hydrogen-bond angles compared to PCs agrees with the ESP difference reported in Figure 1 (d). A blue region is observed in the negative $x$-direction of the amide $\mathrm{C}=\mathrm{O}$ bond, implying a more negative potential using MTPs and hence a stronger interaction of water-hydrogen atoms with the CO-group of NMA.

Table 1: Van der Waals parameters used in the PC and MTP parametrization, respectively. $\epsilon$ is in units of $\mathrm{kJ} / \mathrm{mol}$ and $R_{\text {min }}$ in units of $\AA$.

\begin{tabular}{lrrrr}
\hline \hline & $\epsilon_{\mathrm{PC}}$ & $\epsilon_{\mathrm{MTP}}$ & $R_{\min , \mathrm{PC}}$ & $R_{\min , \mathrm{MTP}}$ \\
\hline $\mathrm{C}$ & -0.46 & -0.46 & 2.00 & 2.10 \\
$\mathrm{CT} 3$ & -0.33 & -0.33 & 2.06 & 2.10 \\
$\mathrm{H}$ & -0.19 & -0.19 & 0.23 & 0.24 \\
$\mathrm{HA}$ & -0.09 & -0.10 & 1.32 & 1.36 \\
$\mathrm{NH}$ & -0.84 & -0.84 & 1.85 & 1.99 \\
$\mathrm{O}$ & -0.50 & -0.50 & 1.70 & 1.78 \\
\hline \hline
\end{tabular}

It may be argued that the change in the solvation shell density is not necessarily due to 
the different (PC and MTP) electrostatics, but could also be due to differences in the vdW interactions, which are optimized separately in the two force fields. The vdW parameters for both models are summarized in Table 1, where the vdW radii in general are larger for the MTP parametrization. Hence, it is expected that the PC parametrization will allow the water molecules to approach NMA more closely compared to the MTP parametrization. In order to quantify this effect, additional MD simulations were carried out, in which the MTP electrostatics was combined with the vdW parameters from the PC force field. The notation MTP/PC will be used for this mixed force field to distinguish it from MTP/MTP and PC/PC where the non-bonded terms (vdW and electrostatics) are jointly optimized.
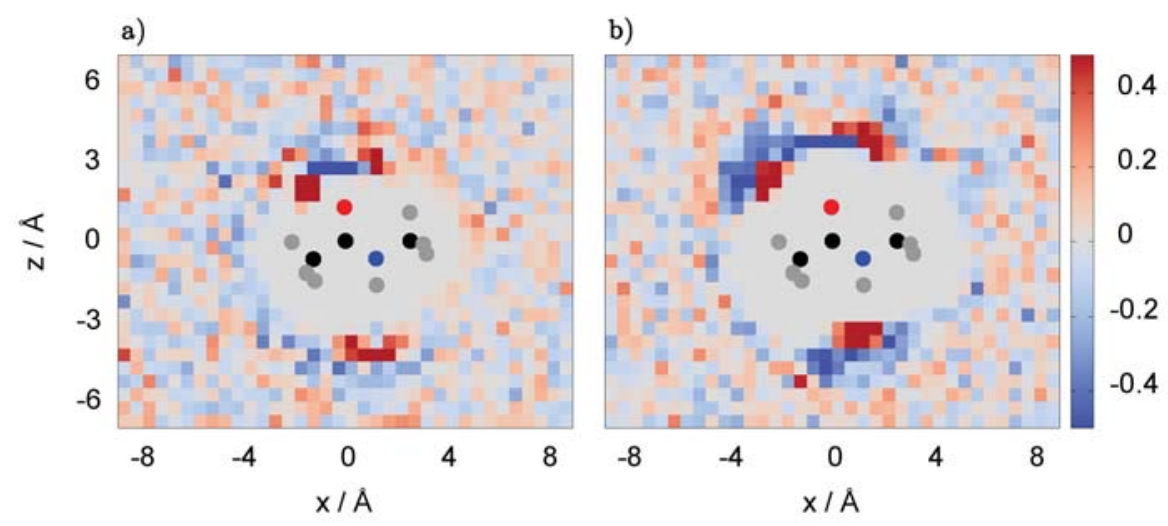

Figure 5: Difference between MTP/PC and PC/PC force fields in the two-dimensional pair distribution function, $g_{\mathrm{MTP} / \mathrm{PC}}-g_{\mathrm{PC} / \mathrm{PC}}$ of a) water hydrogens and b) water oxygens.

Figure 5 displays the difference in water distributions between $\mathrm{MTP} / \mathrm{PC}$ and $\mathrm{PC} / \mathrm{PC}$. The changes in distributions around the amide oxygen between MTP/MTP and PC/PC on the one hand (Figure $3 \mathrm{c}$ ), and $\mathrm{MTP} / \mathrm{PC}$ and $\mathrm{PC} / \mathrm{PC}$ on the other (Figure $5 \mathrm{~b}$ ) are similar. The solvent density for both hydrogens and oxygens in direction of the $\mathrm{C}=\mathrm{O}$ bond is largest using the $\mathrm{PC}$ model (blue regions), while at angles of $\sim 120^{\circ}$ from the bond axis the MTP parametrization leads to a higher density (red regions). This suggests that the difference in solvent distribution around the amide group is caused mainly by the different electrostatic models. Contrarily, the solvent shell around the amide $\mathrm{N}-\mathrm{H}$ group depends on the choice 
of vdW parameters. Both hydrogens and oxygens pack more closely around the solute for the MTP/PC (red regions in Figures 5 (a) and (b)) compared to the MTP/MTP force field (blue regions in Figures 2 (c) and 3 (c)). This can be explained by the smaller vdW radius in the PC parametrization, i.e. $R_{\min , \mathrm{PC}}<R_{\min , \mathrm{MTP}}$ for atom type NH (Tab. 1$)$.

In the following, the solvent dynamics is characterized by analyzing the time scales for different solvent-related processes. They include a) solvent residence times, $\tau_{\text {res }}$, which are determined by counting the average time span a water molecule occupies a given region and are reported in Table $2, \mathrm{~b}$ ) hydrogen-bond fluctuation times $\tau_{\mathrm{H}}$, and c) rotational relaxation times $\tau_{\mathrm{r}, \mathrm{H}}$, both obtained from fitting the decay rate to the corresponding TCF.

Residence times $\left(\tau_{\text {res }}\right)$ for hydrogen-bonded water molecules are calculated as explained in the Method section and listed in Table 2 together with standard deviations $(\sigma)$. The average residence time from simulations with the MTP model is decreased by $\sim 30 \%$ compared to the PC parametrization. Changes in residence lifetimes of this magnitude have potentially important implications for the solvent dynamics and highlight that the electrostatic model employed (PC vs. MTP) directly affects dynamical observables.

Table 2: Residence times with standard deviations for hydrogen bonding or coordination using the PC and MTP parametrization. The relative differences are calculated with respect to the $\mathrm{PC}$ values as $\left(\tau_{\text {res }}^{\mathrm{PC}}-\tau_{\text {res }}^{\mathrm{MTP}}\right) / \tau_{\text {res }}^{\mathrm{PC}}$.

\begin{tabular}{llrrrrr}
\hline \hline & & $\tau_{\text {res }}^{\text {PC }} /$ fs & $\sigma^{\text {PC }} /$ fs & $\tau_{\text {res }}^{\text {MTP }} /$ fs & $\sigma^{\text {MTP }} /$ fs & Rel. diff \\
\hline NMA & $\mathrm{C}=\mathrm{O}$ & 624.8 & 25.4 & 426.2 & 13.5 & $32 \%$ \\
PhBr & Phenyl group & 375.0 & 5.4 & 268.9 & 3.8 & $28 \%$ \\
PhBr & $\sigma$ hole & 250.5 & 2.6 & 220.8 & 5.4 & $12 \%$ \\
\hline \hline
\end{tabular}

In order to further analyze the time evolution of hydrogen bonds we calculate the hydrogenbond fluctuation correlation function, $C_{n}(t)$, as described in the Method section. Figure 6 shows $C_{n}(t)$ for the PC and MTP models, respectively. The decay of $C_{n}(t)$ is well described 


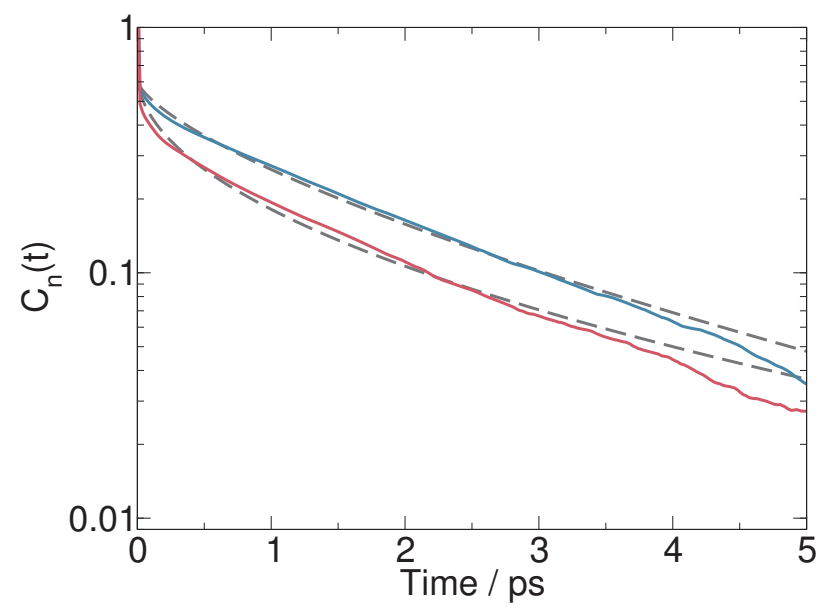

Figure 6: The hydrogen-bond fluctuation correlation function $C_{n}(t)$ of $\delta n(t)$ as a function of time for the PC (blue) and the MTP (red) models, respectively. Dashed grey curves are stretched exponentials fitted to $C_{n}(t)$.

by stretched exponentials of the form $f(t)=A \exp \left[-\left(t / \tau_{\mathrm{H}}\right)^{\beta}\right]$, where $\tau_{\mathrm{H}}$ is the relaxation time and $\beta$ is the stretching exponent. Stretched exponential fits are used to describe relaxation processes, and a value of $\beta=1$ corresponds to a first order decay, while $\beta$-values in the range $[0 ; 1]$ indicate that the decay involves several elementary processes, e.g. due to local traps. ${ }^{56,57}$ By fitting a stretched exponential to $C_{n}(t)$ in the range from 0 to 5 ps we found $\beta=0.69$ for PCs and $\beta=0.50$ for MTPs. The lower $\beta$ value in the MTP system indicates that the solvent relaxes in a more heterogeneous fashion probably as a result of the more anisotropic ESP from the MTP model compared to the PC representation.

The hydrogen-bond fluctuation times from the two fits are $\tau_{\mathrm{H}, \mathrm{PC}}=1.30 \mathrm{ps}$ and $\tau_{\mathrm{H}, \mathrm{MTP}}=0.61$ ps, respectively. Such sub-picosecond decay times are similar to those found in previous work on azide ${ }^{44}$ and $\mathrm{CN}^{-}$from analyzing the same correlation function. ${ }^{30}$ In the case of $\mathrm{CN}^{-}$this time scale coincides with the shortest decay time of the frequency-frequency correlation function (FFCF) and was related to the water librational motion around the solute. For NMAD the FFCF was also determined from three different MTP force fields and the short time scale of its decay ranges from 0.45 to $0.66 \mathrm{ps},{ }^{31}$ depending on the strength of the multipoles 
(in the current work we have used the one with the weakest MTPs). This compares with a longer decay time of $0.91 \mathrm{ps}$ for a PC model. Hence, the fact that a PC model yields slower relaxation of $C_{n}(t)$ than a MTP model is also reflected in the FFCF. Experimentally, the FFCF has been found to involve two time scales with time constants of $0.45 \mathrm{ps}$ and 4.0 ps. ${ }^{49}$ These agree favourably with the results from simulations with a MTP model. This suggests that for hydrogen-bond fluctuation times MTP models outperform PC models.

Further insight into the solvent dynamics can be obtained by considering the rotational reorientation of the solvent in the first solvation shell. Reorientation of water can be separated into two different contributions: A rapid (sub-picosecond) partial reorientation component followed by a slower (picosecond) full reorientation. ${ }^{58}$ The first partial reorientation, also referred to as librations, results from the inertial rotational motion which is hindered by the hydrogen bond network. The subsequent orientational relaxation has been described using different models such as the Debye rotational diffusion and angular Brownian motions. ${ }^{59,60}$

Figure 7 reports the rotational TCF, $C_{r}(t)$, for hydrogen-bonded water. Both PC and MTP parametrizations provide a two-step relaxation of the solvent, where the fast partial reorientation is represented by the local dip in the TCF at $0.02-0.1 \mathrm{ps}$. The long-time relaxation can be described by a single exponential function which has been fitted to $C_{r}(t)$ in the range from 0.15 to 5.0 ps using $f(t)=A \exp \left[-\left(t / \tau_{\mathrm{r}, \mathrm{H}}\right)\right]+B$. Here, the $\mathrm{H}$ subscript denotes that only hydrogen-bonded water molecules are considered (a cut-off $R_{\mathrm{OO}}<3.4 \AA$ between the water and the amide oxygens is used as requirement for the formation of a hydrogen bond, while the hydrogen bond is considered broken when $R_{\mathrm{OO}}>5.0 \AA$ ). Based on a total of 573 hydrogen bonds, this analysis yields average rotational relaxation rates of $\tau_{\mathrm{r}, \mathrm{H}}=1.25 \pm 0.15$ ps using PCs and $\tau_{\mathrm{r}, \mathrm{H}}=1.29 \pm 0.16 \mathrm{ps}$ using MTPs - the difference is not statistically significant. Halle et al. ${ }^{61}$ have studied experimental rotational relaxation times using NMR and reported values in a peptide hydration shell that are slowed down by a factor of 1.2-1.8 


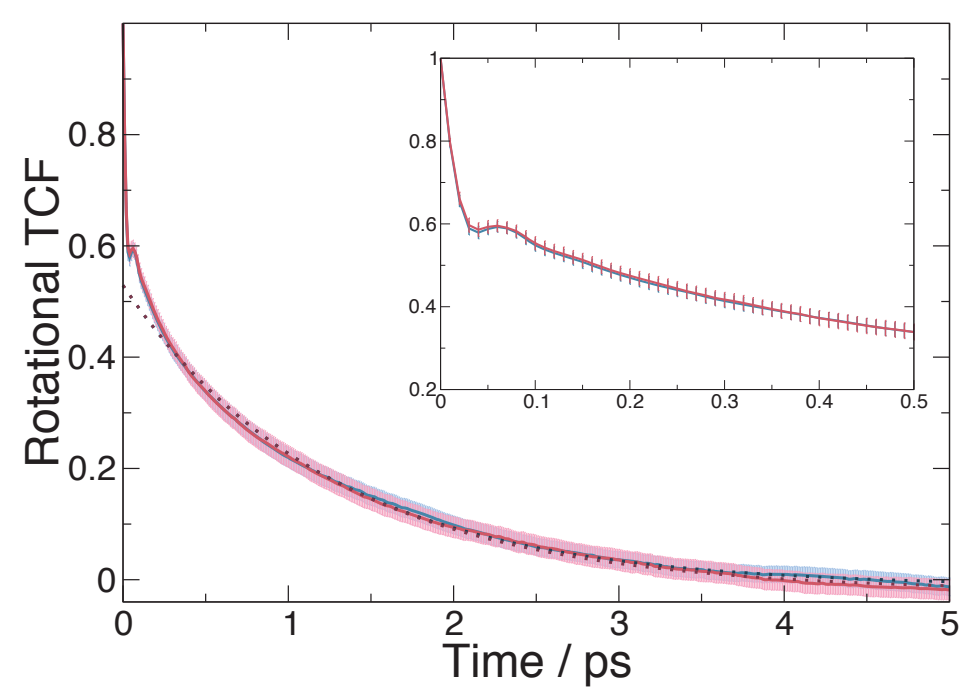

Figure 7: The rotational time correlation function $C_{\mathrm{r}}(t)$ of water molecules hydrogen bonded to the amide oxygen of NMA for the PC (blue) and the MTP (red) models, respectively. The light red and light blue shadows behind the graphs show the standard deviation, while the dotted lines display the exponential fits described in the text. The inset shows a closeup for the first $0.5 \mathrm{ps}$.

compared to bulk water. Based on a 2 ns simulation of 216 TIP3P water molecules, the rotational relaxation time for bulk water is found to be $\tau_{\mathrm{r}, 0}=0.94 \mathrm{ps}$. For the water in the first solvation shell of NMA this corresponds to a slowdown of $\tau_{\mathrm{r}, \mathrm{H}} / \tau_{\mathrm{r}, 0}=1.3$ for PCs and $\tau_{\mathrm{r}, \mathrm{H}} / \tau_{\mathrm{r}, 0}=1.4$ for MTPs, both consistent with the experimental data.

To summarize, we have compared the solvent dynamic results to experimental studies of NMA for both hydrogen-bond fluctuation times ${ }^{49,50}$ and rotational relaxation times, ${ }^{61}$ demonstrating that the MTP force field shows good agreement with experiments, while simulations with a PC model overestimate the hydrogen-bond fluctuation times.

\section{Phenyl bromide}

Halogenated compounds play increasingly important roles in drug design. ${ }^{62-64}$ For correct protein-ligand poses a realistic description of the interaction between the compound and its 


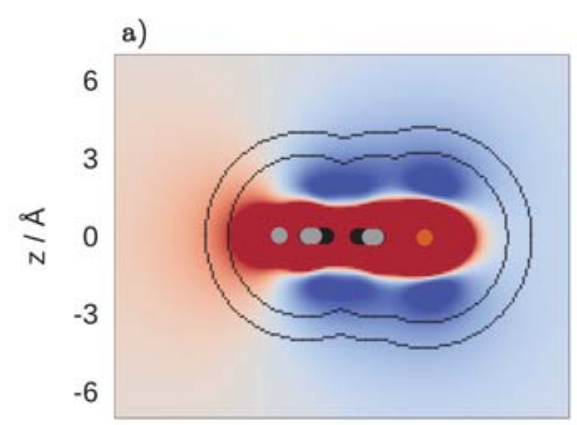

b)

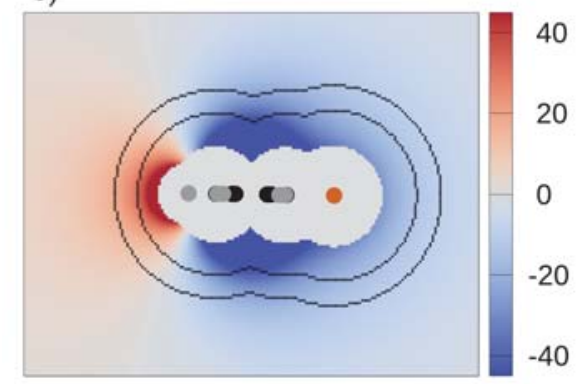

c)

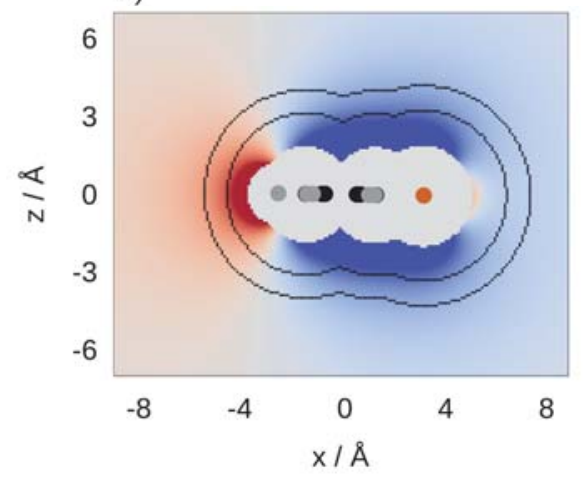

d)

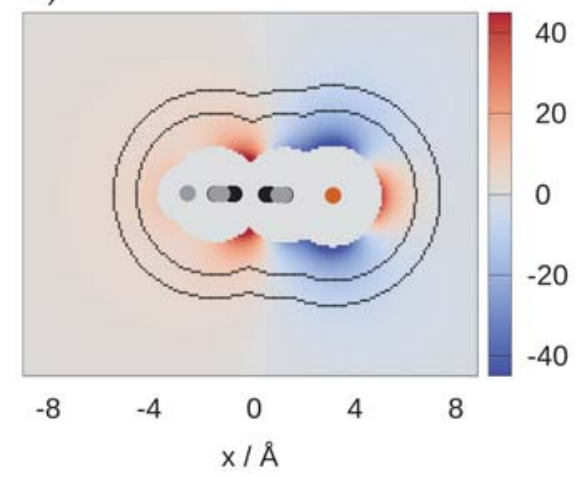

Figure 8: ESP of $\mathrm{PhBr}$ in units of $\mathrm{kJ} / \mathrm{mol}$. a) MP2/cc-pVDZ, b) PC parametrization, c) MTP parametrizations, and d) difference between MTP and PC $\left(\phi_{\mathrm{MTP}}-\phi_{\mathrm{PC}}\right)$. The black contour lines define the region used for fitting the electrostatic parameters, corresponding to $1.66 \sigma$ and $2.2 \sigma$, respectively. 
(nonbonded) environment is required. As a second model system we have therefore studied phenyl bromide $(\mathrm{PhBr})$. The $\pi$-system of the phenyl group is a challenge for PC force fields due to their inability to capture quadrupole moments. Furthermore, atomic PCs are not able to account for the so-called $\sigma$ hole, which is a depletion of electron density along the halogen $\sigma$-bond axis. ${ }^{65,66}$

The ESP around PhBr is calculated at the MP2/cc-pVDZ level and illustrated in Figure 8 (a) on a surface orthogonal to the plane containing the phenyl group and along the $C_{2}$ axis of the system. Figures 8 (b) and 8 (c) report the corresponding ESPs for PCs and MTPs, respectively. The MAD between the two fitted ESPs and the MP2 reference within the fitting region is $5.3 \mathrm{~kJ} / \mathrm{mol}$ for the PC model and $1.3 \mathrm{~kJ} / \mathrm{mol}$ for the MTP model. This demonstrates that the MTP parametrization provides a significant improvement over PCs, in contrast with NMA, for which a different parametrization scheme was applied. The MTP monopoles of NMA were restrained more tightly to their corresponding PC values, compared to the monopoles of $\mathrm{PhBr}$, resulting in a smaller improvement.

Figure 8 (d) shows the difference between MTP- and PC-based ESPs, $\phi_{\mathrm{MTP}}-\phi_{\mathrm{PC}}$, where red regions correspond to the difference between MTP and PC being positive, and vice versa for blue regions. The region along the $\mathrm{C}-\mathrm{Br}$ bond towards the solvent is the $\sigma$ hole and is slightly positive in the MTP model, but slightly negative in the PC model, since atomic PCs cannot account for the strong variability in the ESP.

To analyze the solvent distributions, a local-axis system is defined as described in the Method section. Figure 9 shows the water-hydrogen distributions in the $x z$-plane, while the oxygen distributions are reported in Figure 10. Panels 9 (c) and 10 (c) show the difference between the MTP and PC models, where red (blue) regions correspond to an increased solvent density in the MTP (PC) parametrization. The hydrogen and oxygen distributions show the 


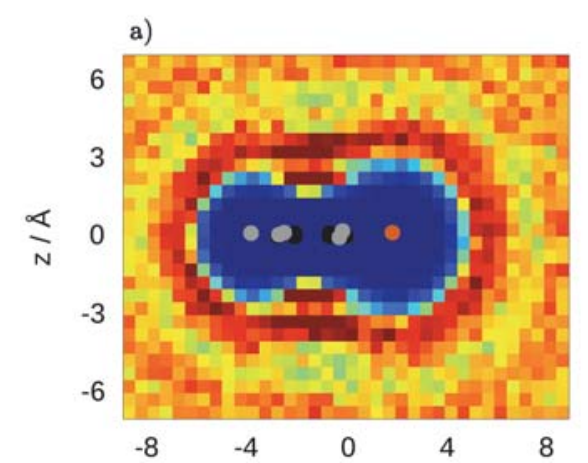

b)

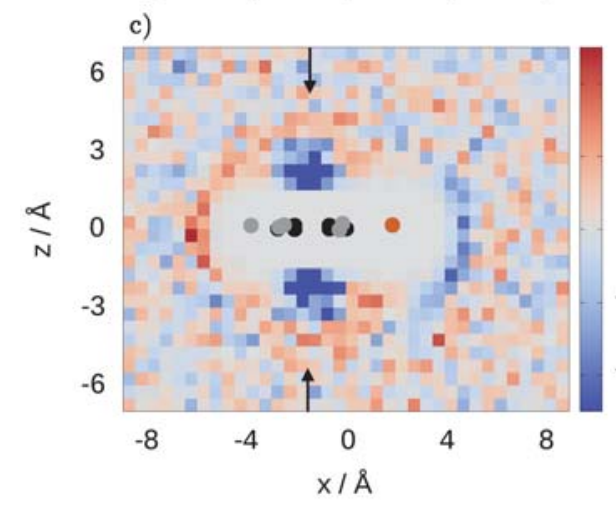

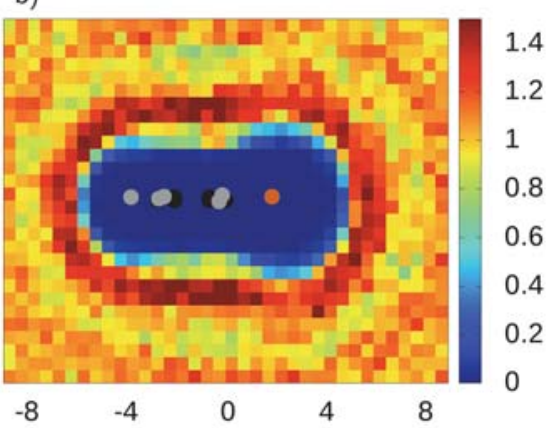

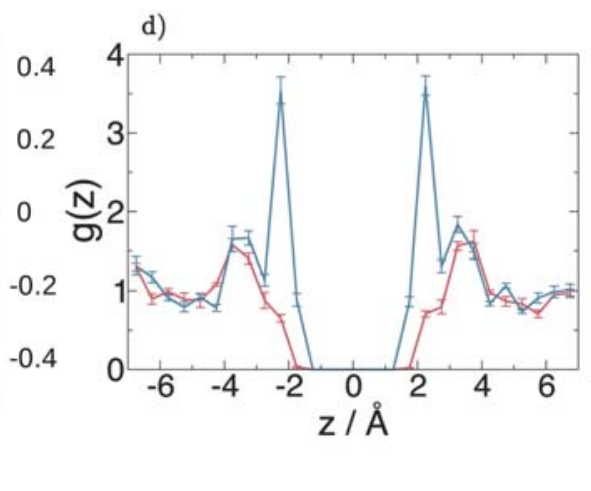

Figure 9: Two-dimensional pair distribution function $g(x, z)$ of water hydrogens plotted along the $C_{2}$-axis of $\mathrm{PhBr}$ and orthogonal to the plane of the phenyl group. Panels a) $\mathrm{PhBr}$ parametrized with PCs and b) parametrized with MTPs. Panels c) difference between MTPs and PCs, $g_{\mathrm{MTP}}-g_{\mathrm{PC}}$, and d) $1 \mathrm{D}$ cut through the centre of the phenyl group $(x=-1.25$ $\AA$ ) with PC shown in blue and MTP in red. The error bars correspond to $1 \sigma$ standard deviations calculated for five independent trajectories. 

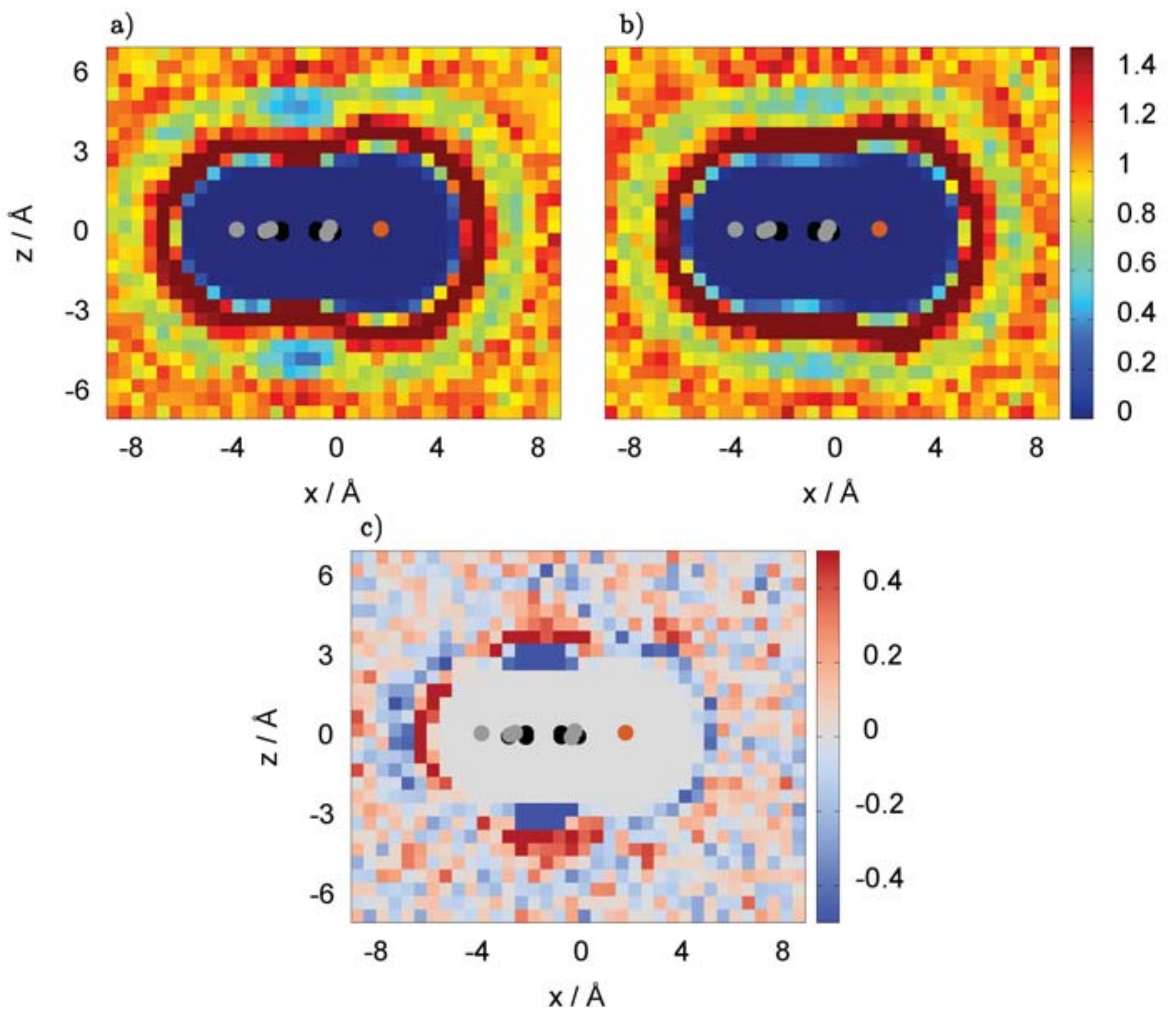

Figure 10: Two-dimensional pair distribution function $g(x, z)$ of water oxygens plotted along the $C_{2}$-axis of $\mathrm{PhBr}$ and orthogonal to the plane of phenyl group. Panels a) $\mathrm{PhBr}$ parametrized with PCs, b) parametrized with MTPs, and c) difference between MTPs and PCs, $g_{\mathrm{MTP}}-g_{\mathrm{PC}}$. 
same trend, but the smaller vdW radius of water hydrogens compared to the water oxygen atoms allows the hydrogens to approach $\mathrm{PhBr}$ closer than the oxygens.

The largest differences in the solvent distribution between the two force fields are found above and below the phenyl plane, where the water (both hydrogens and oxygens) pack more closely around the solute for the PC model compared to the MTP model. The hydrogen distribution as a function of the $z$-coordinate for $x$ and $y$ fixed at the geometrical centre of the phenyl group is given in Figure 9 (d) with error bars reporting the standard deviation. The black arrows in Figure 9 (c) denote this cross section. For PC simulations the first solvation shell is both closer to the solute and also more well-defined in terms of a significantly larger peak. It should be noted that the hydrogen distributions in Figure 9 (d) are very symmetric around $z=0$ ( $\sigma_{v}$ mirror plane), and together with the small standard deviations, this indicates that the simulations (10 ns) are well converged and that the two-dimensional grid is sufficiently large to provide statistically significant pair distribution functions. Standard deviations for Figures 9 and 10 are reported in Figures S3 and S4 in the Supporting Information.

The ESP shown in Figure 8 (d) demonstrates that the potential above and below the phenyl plane is more negative in the PC model than for MTPs, leading to an overestimation of the $\pi \cdots \mathrm{H}-\mathrm{O}$ interaction. Another systematic difference in the solvent distributions is in the region around the $\sigma$ hole, where both hydrogen and oxygen densities are larger using the PC model (blue region in the figures). This is a result of the ESP being slightly positive in the MTP model but negative using PCs. The artificially negative PC potential attracts the water hydrogens and pulls them closer to the solute. The $\sigma$ hole, which is well described in the ESP from a MTP model but missing using PCs, therefore results in a systematic rearrangement of the solvation shell in this region. 
Table 3: Van der Waals parameters for $\mathrm{PhBr}$ used in the PC and MTP parametrization, respectively. $\epsilon$ is in units of $\mathrm{kJ} / \mathrm{mol}$ and $R_{\min }$ in units of $\AA$.

\begin{tabular}{lrrrr}
\hline \hline & $\epsilon_{\mathrm{PC}}$ & $\epsilon_{\mathrm{MTP}}$ & $R_{\text {min,PC }}$ & $R_{\text {min,MTP }}$ \\
\hline $\mathrm{C}$ & -0.21 & -0.33 & 2.00 & 2.00 \\
$\mathrm{H}$ & -0.21 & -0.04 & 1.30 & 1.20 \\
$\mathrm{Br}$ & -2.09 & -1.92 & 2.17 & 2.30 \\
\hline \hline
\end{tabular}

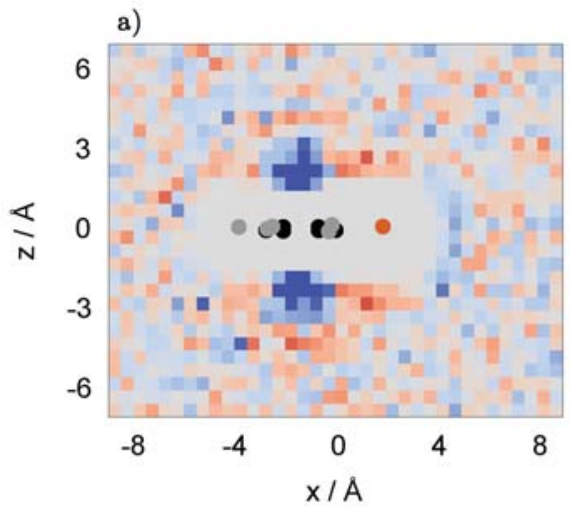

b)

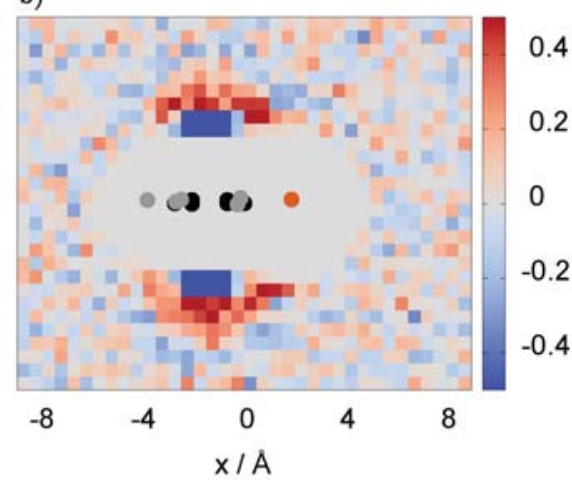

Figure 11: Difference between MTP/PC and PC/PC force fields in the two-dimensional pair distribution function, $g_{\mathrm{MTP} / \mathrm{PC}}-g_{\mathrm{PC} / \mathrm{PC}}$ of a) water hydrogens and b) water oxygens.

Table 3 summarizes the vdW parameters for both force fields, showing that the hydrogen vdW radii are slightly smaller in the MTP model whereas the bromide is larger. To further test the influence of the parametrizations, a 10-ns MD simulation using a mixed MTP/PC force field (i.e., optimized MTPs combined with the vdW parameters of the standard PC force field) were carried out. The differences in water hydrogen and oxygen distributions only due to the use of MTP electrostatics are shown in Figures 11 (a) and 11 (b), and should be compared to Figures 9 (c) and 10 (c), respectively. Above and below the phenyl plane the difference is large and similar to the difference between the fully optimized force fields, illustrating that the differences are not due to the vdW parameters. Around the $\sigma$ - hole, the first solvation shell is more well-defined using the consistent PC/PC compared to the consistent MTP/MTP force field. However, this difference is smaller between the mixed MTP/PC and the consistent $\mathrm{PC} / \mathrm{PC}$ force fields, and only for the hydrogen distribution (blue region at the right-hand side of bromine in Figure 11 (a)). This suggests that the change in solvent 
distributions in this region is partly caused by electrostatics, but also partly by the change in the vdW parameters.

In order to analyze the dynamical effects of the electrostatic model we calculated the residence lifetimes for a water molecule in the two areas corresponding to the largest difference in solvent distribution between the PC and the MTP models, which are the interaction with the $\pi$-system of the phenyl group and in the region of the $\sigma$ hole.

Table 2 summarizes the residence times for the $\mathrm{O}-\mathrm{H} \cdots \pi$ interaction using PCs and MTPs, and shows that $\tau_{\text {res }}$ decreases by $\sim 30 \%$ in the MTP parametrization compared to PC. This is in agreement with the difference in solvent distributions in Figures 9 (c) and (d) and Figure 10 (c), showing that the distribution of solvent using PCs in this region is significantly larger compared to MTPs. Nakahara et. al have studied the dynamics for water in the first solvation shell of benzene using NMR, ${ }^{67}$ which should be very similar to the dynamics in the $\pi$-region of $\mathrm{PhBr}$. They report a correlation time of $\sim 230 \mathrm{fs}$, which agrees well with the residence time of 269 fs using MTPs, and further suggests that the PC simulation overestimate the residence times (375 fs) — the ESP may well be too negative in that region.

The water-residence time around the $\sigma$-hole is determined in the same way. As shown in Table 2, the residence time of a water oxygen in this region of space is $\sim 10 \%$ smaller for PhBr parametrized with MTPs compared to the PC model. The shorter $\tau_{\text {res }}$ with a MTP parametrization is unexpected since PC electrostatics is not suitable to describe halogen bonding, but this will be investigated separately. 


\section{Summary and Conclusion}

The present work provides a systematic investigation of the importance of higher order MTPs for equilibrium and dynamical properties in condensed-phase simulations. Solute-solvent interactions using both MTP and PC electrostatics have been analyzed in regions of hydrogen bonding, $\pi$-orbitals, and halogen interactions, where commonly used PC force fields give inaccurate representations of the ESP due to their lack of anisotropy. ${ }^{68-74}$

For NMA, direct comparison with experimental data is possible. The hydrogen-bond fluctuation times and rotational relaxation times computed from the MTP FF show good agreement with experiments, while simulations with a PC model overestimate the hydrogen-bond fluctuation times. For PhBr the ESPs of the PC and MTP models differ by $5.3 \mathrm{~kJ} / \mathrm{mol}$ and $1.3 \mathrm{~kJ} / \mathrm{mol}$ from the reference MP2 calculations demonstrating that the MTP parametrization provides a significant improvement over PCs. Direct comparison of the solvent dynamics around $\mathrm{PhBr}$ with experiment is not possible due to lack of explicit measurements. However, the water residence times around benzene agree to within less than $10 \%$ when analyzing the relevant regions around $\mathrm{PhBr}$ from simulations with MTPs and differ by more than 70 \% when PCs are used.

Considering dynamical quantities for which explicit experimental data is available one finds that the MTP FF provides a more realistic description of hydrogen-bond structures, while the PC model leads to larger deviations in the geometry. The many experimental studies of NMA allow a more thorough validation of the dynamical properties, and it is concluded that in this case the MTP FF shows good agreement with the available experiments throughout the present study wherever experimental data for direct comparison is available.

Residence lifetimes have been calculated for three different solvent-solute coordinations (hydrogen bonding, $\pi \cdots \mathrm{H}-\mathrm{O}$, and $\sigma \cdots \mathrm{O}-\mathrm{H}$ ), resulting in variations between the FFs of 
$10-30 \%$. Relaxation times of hydrogen-bonded waters differ by a factor of two, where the MTP simulations better match experimental results. It is evident that the electrostatic model has large implications on the solvent dynamics, and variations of this magnitude will significantly influence, for example, the diffusion of a ligand in and out of the protein binding site. ${ }^{75,76}$

Beyond the improved dynamic behavior observed using MTP force fields, which is also observed previously for various molecules, ${ }^{24,28,30,31}$ it is the systematic gain from MTPs that we find across molecules, chemical environments, and force-field parametrization strategies that make for the most compelling conclusion. This improvement does not require altering of the solvent force field and comes at small additional computational cost, since the MTPs are added on the solute molecule only.

\section{Supporting Information}

Standard deviations for the solvent distribution plots for NMA (Figures 2 and 3 in the paper) and $\mathrm{PhBr}$ (Figures 9 and 10 in the paper) are provided as Supporting Information. This material is available free of charge via the internet at http://pubs.acs.org

\section{Acknowledgments}

The authors gratefully acknowledge financial support from the Swiss National Science Foundation through grant 200021-117810 and to the NCCR-MUST. Support from the Danish Center for Scientific Computation and the Danish Natural Science Research Council is gratefully acknowledged. 


\section{References}

(1) Jensen, F. Introduction to Computational Chemistry, 2nd ed.; Wiley, 2007.

(2) Rick, S. W.; Stuart, S. J. Reviews in Computational Chemistry; John Wiley and Sons, Inc., 2003; Vol. 18; Chapter 3, pp 89-146.

(3) Nutt, D. R.; Meuwly, M. Theoretical Investigation of Infrared Spectra and Pocket Dynamics of Photodissociated Carbonmonoxy Myoglobin. Biophys. J. 2003, 85, 36123623.

(4) Halgren, T. A.; Damm, W. Polarizable Force Fields. Curr. Op. in Struct. Biol. 2001, $11,236-242$.

(5) Shi, Y.; Xia, Z.; Zhang, J.; Best, R.; Wu, C.; Ponder, J. W.; Ren, P. Polarizable Atomic Multipole-Based AMOEBA Force Field for Proteins. J. Chem. Theory Comput. 2013, 9, 4046-4063.

(6) Lopes, P. E. M.; Huang, J.; Shim, J.; Luo, Y.; Li, H.; Roux, B.; MacKerell, A. D. Polarizable Force Field for Peptides and Proteins Based on the Classical Drude Oscillator. J. Chem. Theory Comput. 2013, 9, 5430-5449.

(7) Beck, B.; Rauhut, G.; Clark, T. The Natural Atomic Orbital Point Charge Model for PM3: Multipole Moments and Molecular Electrostatic Potentials. J. Comp. Chem. 1994, 15, 1064-1073.

(8) Ritchie, J. P.; Copenhaver, A. S. Comparison of Potential-Derived Charge and Atomic Multipole Models in Calculating Electrostatic Potentials and Energies of Some Nucleic Acid Bases and Pairs. J. Comp. Chem. 1995, 16, 777-789.

(9) Gedeck, P.; Schindler, T.; Alex, A.; Clark, T. New Multicentre Point Charge Models for Molecular Electrostatic Potentials from Semiempirical M0-Calculations. J. Mol. Model. 2000, 6, 452-466. 
(10) Le, H.-A.; Lee, A. M.; Bettens, R. P. A. Accurately Reproducing Ab Initio Electrostatic Potentials with Multipoles and Fragmentation. J. Phys. Chem. A 2009, 113, 1052710533.

(11) Kramer, C.; Gedeck, P.; Meuwly, M. Atomic Multipoles: Electrostatic Potential Fit, Local Reference Axis Systems and Conformational Dependence. J. Comp. Chem. 2012, 33, 1673-1688.

(12) Jakobsen, S.; Kristensen, K.; Jensen, F. Electrostatic Potential of Insulin: Exploring the Limitations of Density Functional Theory and Force Field Methods. J. Chem. Theory Comput. 2013, 9, 3978-3985.

(13) Cardamone, S.; Hughes, T. J.; Popelier, P. L. A. Multipolar Electrostatics. Phys. Chem. Chem. Phys. 2014, 16, 10367-10387.

(14) Jorgensen, W. L.; Schyman, P. Treatment of Halogen Bonding in the OPLS-AA Force Field: Application to Potent Anti-HIV Agents. Journal of Chemical Theory and Computation 2012, 8, 3895-3901.

(15) Mahoney, M. W.; Jorgensen, W. L. A Five-Site Model for Liquid Water and the Reproduction of the Density Anomaly by Rigid, Nonpolarizable Potential Functions. J. Chem. Phys. 2000, 112, 8910-8922.

(16) Francl, M. M.; Carey, C.; Chirlian, L. E.; Gange, D. M. Charges Fit to Electrostatic Potentials. II. Can Atomic Charges be Unambiguously Fit to Electrostatic Potentials? J. Comp. Chem. 1996, 17, 367-383.

(17) Francl, M. M.; Chirlian, L. E. Rev. Comput. Chem.; John Wiley and Sons, Inc., 2000; Vol. 14; Chapter 1, pp 1-31.

(18) Stone, A. J. The Theory of Intermolecular Forces; Oxford University Press, 2002. 
(19) Stone, A. J.; Alderton, M. Distributed Multipole Analysis: Methods and Applications. Molecular Physics 1985, 100, 221-233.

(20) Stone, A. J. Distributed Multipole Analysis: Stability for Large Basis Sets. J. Chem. Theo. Comp. 2005, 1, 1128-1132.

(21) Sokalski, W.; Poirier, R. Cumulative Atomic Multipole Representation of the Molecular Charge Distribution and its Basis Set Dependence. Chem. Phys. Lett. 1983, 98, 86-92.

(22) Sokalski, W. A.; Sawaryn, A. Correlated Molecular and Cumulative Atomic Multipole Moments. J. Chem. Phys. 1987, 87, 526-534.

(23) Bader, R. F. W. Atoms in Molecules. A Quantum Theory; Oxford Univ. Press: Oxford, Great Britain, 1990.

(24) Ponder, J. W.; Wu, C.; Ren, P.; Pande, V. S.; Chodera, J. D.; Schnieders, M. J.; Haque, I.; Mobley, D. L.; Lambrecht, D. S.; DiStasio, R. A.; et al., Current Status of the AMOEBA Polarizable Force Field. J. Phys. Chem. B 2010, 114, 2549-2564.

(25) Rasmussen, T. D.; Ren, P.; Ponder, J. W.; Jensen, F. Force Field Modeling of Conformational Energies: Importance of Multipole Moments and Intramolecular Polarization. Int. J. Quantum Chem. 2007, 107, 1390-1395.

(26) Bereau, T.; Kramer, C.; Monnard, F. W.; Nogueira, E. S.; Ward, T. R.; Meuwly, M. Scoring Multipole Electrostatics in Condensed-Phase Atomistic Simulations. J. Phys. Chem. B 2013, 117, 5460-5471.

(27) Kramer, C.; Gedeck, P.; Meuwly, M. Multipole-Based Force Fields from ab Initio Interaction Energies and the Need for Jointly Refitting All Intermolecular Parameters. J. Chem. Theo. Comp. 2013, 9, 1499-1511.

(28) Plattner, N.; Meuwly, M. Quantifying the Importance of Protein Conformation on Ligand Migration in Myoglobin. Biophys. J. 2012, 102, 333-341. 
(29) Lee, M. W.; Meuwly, M. On the Role of Nonbonded Interactions in Vibrational Energy Relaxation of Cyanide in Water. J. Phys. Chem. A 2011, 115, 5053-5061.

(30) Lee, M. W.; Carr, J. K.; Göllner, M.; Hamm, P.; Meuwly, M. 2D IR Spectra of Cyanide in Water Investigated by Molecular Dynamics Simulations. J. Chem. Phys. 2013, 139, 054506 .

(31) Cazade, P.-A.; Bereau, T.; Meuwly, M. Computational Two-Dimensional Infrared Spectroscopy without Maps: N-Methylacetamide in Water. J. Phys. Chem. B 2014, 118, $8135-8147$.

(32) Lee, M. W.; Meuwly, M. Hydration Free Energies of Cyanide and Hydroxide Ions from Molecular Dynamics Simulations with Accurate Force Fields. Phys. Chem. Chem. Phys. 2013, 15, 20303-20312.

(33) Kramer, C.; Bereau, T.; Spinn, A.; Liedl, K. R.; Gedeck, P.; Meuwly, M. Deriving Static Atomic Multipoles from the Electrostatic Potential. J. Chem. Inf. Model. 2013, $53,3410-3417$.

(34) Vanommeslaeghe, K.; MacKerell, A. D. Automation of the CHARMM General Force Field (CGenFF) I: Bond Perception and Atom Typing. J. Chem. Inf. Model. 2012, 52, $3144-3154$.

(35) Vanommeslaeghe, K.; Raman, E. P.; MacKerell, A. D. Automation of the CHARMM General Force Field (CGenFF) II: Assignment of Bonded Parameters and Partial Atomic Charges. J. Chem. Inf. Model. 2012, 52, 3155-3168.

(36) Bereau, T.; Kramer, C.; Meuwly, M. Leveraging Symmetries of Static Atomic Multipole Electrostatics in Molecular Dynamics Simulations. J. Chem. Theory Comput. 2013, 9, 5450-5459. 
(37) Jorgensen, W. L.; Chandrasekhar, J.; Madura, J. D.; Impey, R. W.; Klein, M. L. Comparison of Simple Potential Functions for Simulating Liquid Water. J. Chem. Phys. 1983, $79,926-935$.

(38) Brooks, B. R. et al. CHARMM: The Biomolecular Simulation Program. J. Comp. Chem. 2009, 30, 1545-1614.

(39) Feller, S. E.; Zhang, Y.; Pastor, R. W.; Brooks, B. R. Constant Pressure Molecular Dynamics Simulation: The Langevin Piston Method. J. Chem. Phys. 1995, 103, 4613.

(40) Luzar, A.; Chandler, D. Structure and Hydrogen Bond Dynamics of Water-Dimethyl Sulfoxide Mixtures by Computer Simulations. J. Chem. Phys. 1993, 98, 8160-8173.

(41) Prakash, M.; Samy, K. G.; Subramanian, V. Benzene-Water (BZWn (n = 1 - 10)) Clusters. J. Phys. Chem. A 2009, 113, 13845-13852.

(42) Guo, H.; Karplus, M. Ab Initio Studies of Hydrogen Bonding of N-Methylacetamide: Structure, Cooperativity, and Internal Rotational Barriers. J. Phys. Chem. 1992, 96, $7273-7287$.

(43) Yang, S.; Cho, M. IR Spectra of N-Methylacetamide in Water Predicted by Combined Quantum Mechanical/Molecular Mechanical Molecular Dynamics Simulations. J. Chem. Phys. 2005, 123, 134503.

(44) Li, S.; Schmidt, J. R.; Corcelli, S. A.; Lawrence, C. P.; Skinner, J. L. Approaches for the Calculation of Vibrational Frequencies in Liquids: Comparison to Benchmarks for Azide/Water Clusters. J. Chem. Phys. 2006, 124, 204110.

(45) Bastida, A.; Soler, M. A.; Zuniga, J.; Requena, A.; Kalstein, A.; Fernandez-Alberti, S. Hybrid Quantum/Classical Simulations of the Vibrational Relaxation of the Amide I Mode of N-Methylacetamide in $\mathrm{D}_{2} \mathrm{O}$ Solution. J. Phys. Chem. B 2012, 116, 2969-2980. 
(46) Farag, M. H.; Bastida, A.; Ruiz-López, M. F.; Monard, G.; Ingrosso, F. Vibrational Energy Relaxation of the Amide I Mode of N-Methylacetamide in $\mathrm{D}_{2} \mathrm{O}$ Studied through Born-Oppenheimer Molecular Dynamics. J. Phys. Chem. B 2014, 118, 6186-6197.

(47) Barth, A. Infrared Spectroscopy of Proteins. Biochim. Biophys. Acta, Bioenerg. 2007, $1767,1073-1101$.

(48) Zanni, M. T.; Asplund, M. C.; Hochstrasser, R. M. Two-Dimensional Heterodyned and Stimulated Infrared Photon Echoes of N-Methylacetamide-D. J. Chem. Phys. 2001, 114, 4579-4590.

(49) Hamm, P.; Lim, M.; Hochstrasser, R. M. Structure of the Amide I Band of Peptides Measured by Femtosecond Nonlinear-Infrared Spectroscopy. J. Phys. Chem. B 1998, 102, 6123-6138.

(50) Woutersen, S.; Pfister, R.; Hamm, P.; Mu, Y.; Kosov, D. S.; Stock, G. Peptide Conformational Heterogeneity Revealed from Nonlinear Vibrational Spectroscopy and Molecular-Dynamics Simulations. J. Chem. Phys. 2002, 117, 6833-6840.

(51) Bastida, A.; Soler, M. A.; Zuniga, J.; Requena, A.; Kalstein, A.; Fernandez-Alberti, S. Instantaneous Normal Modes, Resonances, and Decay Channels in the Vvibrational Relaxation of the Amide I Mode of N-Methylacetamide-D in Liquid Deuterated Water. J. Chem. Phys. 2010, 132, 224501.

(52) MacKerell, A. D. In Encyclopedia of Computational Chemistry; Schleyer, P. v. R., Ed.; John Wiley and Sons, 2002; Vol. 3; pp 2191-2200.

(53) Price, D. J.; Brooks, C. L. Modern Protein Force Fields Behave Comparably in Molecular Dynamics Simulations. J. Comp. Chem. 2002, 23, 1045-1057.

(54) Mannfors, B.; Mirkin, N. G.; Palmo, K.; Krimm, S. A Polarizable Electrostatic Model of the N-Methylacetamide Dimer. J. Comp. Chem. 2001, 22, 1933-1943. 
(55) Yang, Z.-Z.; Qian, P. A Study of N-Methylacetamide in Water Clusters: Based on Atom-Bond Electronegativity Equalization Method Fused into Molecular Mechanics. J. Chem. Phys. 2006, 125.

(56) Phillips, J. C. Stretched Exponential Relaxation in Molecular and Electronic Glasses. Rep. Prog. Phys. 1996, 59, 1133-1207.

(57) Mattson, J.; Wyss, H. M.; Fernandez-Nieves, A.; Miyazaki, K.; Hu, Z.; Reichman, D. R.; Weitz, D. A. Soft Colloids Make Strong Glasses. Nature 2009, 462, 83-86.

(58) Laage, D.; Stirnemann, G.; Sterpone, F.; Rey, R.; Hynes, J. T. Reorientation and Allied Dynamics in Water and Aqueous Solutions. Annu. Rev. Phys. Chem. 2011, 62, $395-416$.

(59) Gordon, R. G. On the Rotational Diffusion of Molecules. J. Chem. Phys. 1966, 44, $1830-1836$.

(60) Furry, W. Isotropic Rotational Brownian Motion. Phys. Rev. 1957, 10\%, 7-13.

(61) Qvist, J.; Persson, E.; Mattea, C.; Halle, B. Time Scales of Water Dynamics at Biological Interfaces: Peptides, Proteins and Cells. Faraday Discuss. 2009, 141, 131-144.

(62) Auffinger, P.; Hays, F. A.; Westhof, E.; Ho, P. S. Halogen Bonds in Biological Molecules. Proc. Natl. Acad. Sci. U. S. A. 2004, 101, 16789-16794.

(63) Müller, K.; Faeh, C.; Diederich, F. Fluorine in Pharmaceuticals: Looking Beyond Intuition. Science 2007, 317, 1881-1886.

(64) Hardegger, L. A.; Kuhn, B.; Spinnler, B.; Anselm, L.; Ecabert, R.; Stihle, M.; Gsell, B.; Thoma, R.; Diez, J.; Benz, J.; et al., Systematic Investigation of Halogen Bonding in Protein-Ligand Interactions. Ang. Chem. Int. Ed. 2011, 50, 314-318.

(65) Clark, T.; Hennemann, M.; Murray, J.; Politzer, P. Halogen Bonding: The $\sigma$-Hole. J. Mol. Model. 2007, 13, 291-296. 
(66) Politzer, P.; Murray, J. S.; Lane, P. $\sigma$-Hole Bonding and Hydrogen Bonding: Competitive Interactions. Int. J. Quantum Chem. 2007, 10\%, 3046-3052.

(67) Nakahara, M.; Wakai, C.; Yoshimoto, Y.; Matubayasi, N. Dynamics of Hydrophobic Hydration of Benzene. J. Phys. Chem. 1996, 100, 1345-1349.

(68) Freddolino, P. L.; Harrison, C. B.; Liu, Y.; Schulten, K. Challenges in Protein-Folding Simulations. Nat. Phys. 2010, 6, 751-758.

(69) Lu, Z.; Zhou, N.; Wu, Q.; Zhang, Y. Directional Dependence of Hydrogen Bonds: A Density-Based Energy Decomposition Analysis and Its Implications on Force Field Development. J. Chem. Theory Comput. 2011, 7, 4038-4049.

(70) Best, R. B.; Hummer, G. Optimized Molecular Dynamics Force Fields Applied to the HelixâĽ̌Coil Transition of Polypeptides. J. Phys. Chem. B 2009, 113, 9004-9015.

(71) Kortemme, T.; Morozov, A. V.; Baker, D. An Orientation-Dependent Hydrogen Bonding Potential Improves Prediction of Specificity and Structure for Proteins and ProteinProtein Complexes. J. Mol. Biol. 2003, 326, 1239-1259.

(72) Caldwell, J. W.; Kollman, P. A. Cation- $\pi$ Interactions: Nonadditive Effects Are Critical in Their Accurate Representation. J. Am. Chem. Soc. 1995, 117, 4177-4178.

(73) Politzer, P.; Lane, P.; Concha, M.; Ma, Y.; Murray, J. An Overview of Halogen Bonding. Journal of Molecular Modeling 2007, 13, 305-311.

(74) Politzer, P.; Murray, J. S.; Concha, M. C. $\sigma$-Hole Bonding Between Like Atoms; a Fallacy of Atomic Charges. J. Mol. Model. 2008, 14, 659-665.

(75) Buch, I.; Giorgino, T.; De Fabritiis, G. Complete Reconstruction of an EnzymeInhibitor Binding Process by Molecular Dynamics Simulations. Proc. Natl. Acad. Sci. 2011, 108, 10184-10189. 
(76) Dror, R. O.; Pan, A. C.; Arlow, D. H.; Borhani, D. W.; Maragakis, P.; Shan, Y.; Xu, H.; Shaw, D. E. Pathway and Mechanism of Drug Binding to G-Protein-Coupled Receptors. Proc. Natl. Acad. Sci. 2011, 13118-13123. 


\section{For Table of Contents Use Only}

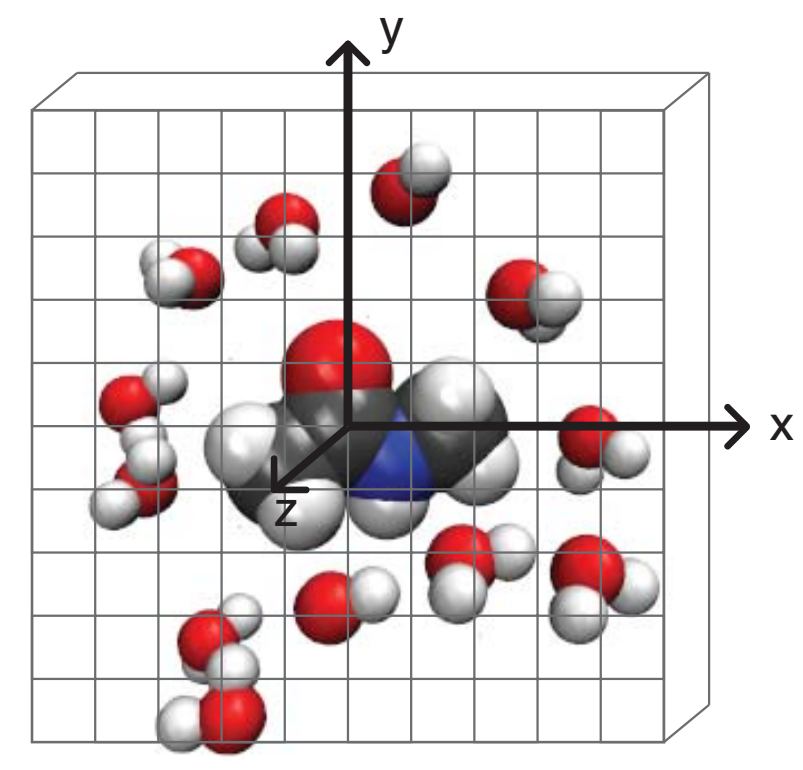

